\title{
Self-Rated Health in middle-aged and elderly Chinese: distribution, determinants and associations with cardio-metabolic risk factors
}

\author{
Nazanin Haseli-Mashhadi ${ }^{1}$, An Pan ${ }^{2}$, Xingwang Ye², Jing Wang2, Qibin Qi², \\ Yong Liu ${ }^{2}$, Huaixing $\mathrm{Li}^{2}$, Zhijie $\mathrm{Yu}^{2}, \mathrm{Xu} \mathrm{Lin}^{2}$ and Oscar H Franco*1,3
}

Address: ${ }^{1}$ Unilever R\&D, Colworth Science Park, Sharnbrook, Bedfordshire, MK441LQ, UK, ${ }^{2}$ Key Laboratory of Nutrition and Metabolism, Institute for Nutritional Sciences, Shanghai Institutes for Biological Sciences, Chinese Academy of Sciences and Graduate School of the Chinese Academy of Sciences, Shanghai, PR China and ${ }^{3}$ Health Sciences Research Institute, Warwick Medical School, The University of Warwick. Coventry CV47AL, UK

Email: Nazanin Haseli-Mashhadi - Nazanin.Haseli_Mashhadi@unilever.com; An Pan - apan@sibs.ac.cn; Xingwang Ye - xwye@sibs.ac.cn; Jing Wang - wangjing@sibs.ac.cn; Qibin Qi - zjyu@sibs.ac.cn; Yong Liu - xlin@sibs.ac.cn; Huaixing Li - huaixing@hotmail.com; ZhijieYu - zjyu@sibs.ac.cn; Xu Lin - xlin@sibs.ac.cn; Oscar H Franco* - o.H.Franco@warwick.ac.uk

* Corresponding author

Published: 29 September 2009

BMC Public Health 2009, 9:368 doi:10.1 186/147/-2458-9-368
Received: 6 November 2008

Accepted: 29 September 2009

This article is available from: http://www.biomedcentral.com/147I-2458/9/368

(c) 2009 Haseli-Mashhadi et al; licensee BioMed Central Ltd.

This is an Open Access article distributed under the terms of the Creative Commons Attribution License (http://creativecommons.org/licenses/by/2.0), which permits unrestricted use, distribution, and reproduction in any medium, provided the original work is properly cited.

\begin{abstract}
Background: Self-rated health (SRH) has been demonstrated to be an accurate reflection of a person's health and a valid predictor of incident mortality and chronic morbidity. We aimed to evaluate the distribution and factors associated with SRH and its association with biomarkers of cardio-metabolic diseases among middle-aged and elderly Chinese.
\end{abstract}

Methods: Survey of I,458 men and I,83I women aged 50 to 70 years, conducted in one urban and two rural areas of Beijing and Shanghai in 2005. SRH status was measured and categorized as good (very good and good) vs. not good (fair, poor and very poor). Determinants of SRH and associations with biomarkers of cardio-metabolic diseases were evaluated using logistic regression.

Results: Thirty two percent of participants reported good SRH. Males and rural residents tended to report good SRH. After adjusting for potential confounders, residence, physical activity, employment status, sleep quality and presence of diabetes, cardiovascular disease, and depression were the main determinants of SRH. Those free from cardiovascular disease $(\mathrm{OR} 3.68 ; 95 \% \mathrm{Cl} 2.39$; 5.66), rural residents (OR I.89; $95 \% \mathrm{Cl}$ I.47; 2.43), non-depressed participants (OR 2.50; $95 \% \mathrm{Cl}$ I.67; 3.73 ) and those with good sleep quality (OR $2.95 ; 95 \% \mathrm{Cl} 2.22 ; 3.91$ ) had almost twice or over the chance of reporting good SRH compared to their counterparts. There were significant associations -and trend- between SRH and levels of inflammatory markers, insulin levels and insulin resistance.

Conclusion: Only one third of middle-aged and elderly Chinese assessed their health status as good or very good. Although further longitudinal studies are required to confirm our findings, interventions targeting social inequalities, lifestyle patterns might not only contribute to prevent chronic morbidity but as well to improve populations' perceived health. 


\section{Background}

Self-rated health (SRH) is a simple but comprehensive self-assessment of global (physical and mental) health status in which respondents are asked to rate their overall health, usually in a 5-point scale [1-3]. In previous studies, in a wide variety of populations, SRH has been demonstrated to be an accurate reflection of a person's health and a valid predictor of incident mortality and chronic morbidity (e.g. depression, diabetes) [3-10] that might be just as good as collecting extensive biological data [3,9]. Yu et al. [10] found that poor perceived health increased the relative risk of death by almost twice compared to excellent/good health among urban Shanghai residents. Leung et al. [8] studying elderly Chinese found a six time higher relative risk of death for those with poor levels of SRH (compared to good/excellent). Nevertheless, comprehensive assessments of the determinants of SRH among Chinese populations are scarce. Only a few studies [2,11-14] have evaluated the levels of SRH among Chinese but they only consider a single demographic nucleus, ignoring the potential effect of geographic location and residential status in a vast and populous country like China. Other factors such as sleep patterns and depression which might also significantly influence SRH have not been extensively investigated in the existing studies $[2,11$ 14]. Furthermore just a few studies [15-17], none in mainland China, have evaluated whether SRH besides being a predictor of mortality and morbidity, could be associated with biomarkers of cardio-metabolic disorders among healthy populations and thus reflect early deviations from healthy trajectories.

We aimed to evaluate: 1) the distribution of SRH, among middle-aged and elderly populations in China; 2 ) the factors that determine SRH in this population; and 3) whether lower SRH is associated with worse levels of biomarkers of cardio-metabolic disease.

\section{Methods}

\section{Study population}

The current study is part of the "Nutrition and Health of Aging Population in China" study [18]. To provide a representative sample of elderly people in China, a cross-sectional survey of 3289 subjects $(1,458$ men and 1,831 women) aged 50 to 70 years from one urban and two rural areas of Beijing and Shanghai (cities of north and south of China) was conducted from March to June 2005. Streets or villages were randomly selected. Then participants were randomly selected from the eligible candidates listed in the residential registration record who were stable residents for 20 years in the area and were free from the following condition: 1) severe psychological disorders, physical disabilities, cancer, CVD, Alzheimer's disease or dementia, within 6 months; or 2) currently diagnosed with tuberculosis, AIDS and other communicable disease.
The sampling was conducted with an emphasis on enlisting at least $40 \%$ of men, a similar number of rural/urban residents of Beijing and Shanghai and with representation from all levels of education and income. From a residency registration list in the selected streets or villages, 3533 potential participants were identified and interviewed in their households. The total response rate was $93.1 \%$ with no difference between the cities (93.5\% in Beijing and $92.7 \%$ in Shanghai). A face to face interview with one respondent per household was completed by trained physicians or public health workers using a standardized questionnaire. 3379 persons agreed to participate in the study while 3289 eligible participants with complete information from questionnaire, physical examination and biomarkers were included in the final analysis. Selfrated health status and extensive socio-demographical, lifestyle and chronic disease information were collected from the participants. The study was approved by the Institutional Review Board of Institute for Nutrition Sciences, and informed consent was obtained from each participant. Further details about the questionnaire can be found elsewhere [19].

SRH was initially recorded in 5 levels (very good, good, fair, poor, and very poor), and then categorized into two categories good (very good and good) and poor (fair, poor and very poor) $[2,13]$.

Socio-demographic variables included age (categorized as 50-59 and 60-70 yrs), gender, geographic region (Beijing/ Shanghai), residential area (urban/rural), marital status (defined as married or without spouse (single, separated or widow)) and living status (defined as living alone versus with others). Socio-economic variables included educational attainment (categorized into low, moderate and high, based on the number of years of education as 0-6, 79 , and $\geq 10$ years respectively), employment status (grouped as currently employed, unemployed/on welfare system/retired) and total household income (categorized as low, moderate and high on the basis of $<10,000$, $10,000-29,999$ and $>=30,000$ RMB annually).

Lifestyle variables included social activity (defined as "yes"/"no" on the basis of frequently participating in at least one of social activities such as painting, playing chess, singing and dancing), smoking habit [defined as never, current (daily smoking, $>6$ months) and former (cessation of smoking $>6$ months)]; alcohol drinking ("yes "/"no"); physical activity level (classified as low, moderate, or high according to the guidelines for data processing and analysis of the International Physical Activity Questionnaire $[5,20]$ ); sleep quality during the last month (well, common or poor); and sleep quantity: average total hours of daily sleep during last month (cate- 
gorized as under, normal and over $(<7,7$ to 9 , and $>9$ hours per day, respectively)).

Body mass index (BMI) levels were categorized as underweight $(<18.5 \mathrm{~kg} / \mathrm{m} 2)$, normal weight $(18.5$ to $<24.0 \mathrm{~kg} /$ $\mathrm{m} 2$ ), overweight ( 24.0 to $<28.0 \mathrm{~kg} / \mathrm{m} 2)$ and obese ( $\geq 28.0$ $\mathrm{kg} / \mathrm{m} 2)[21]$.

Medical insurance defined as "yes"/"no" on the basis of benefiting from one type of medical insurance such as social, commercial or governmental paid.

Three measurements of blood pressure were taken using an electronic blood pressure monitor and the mean of the last two measurements was used for analysis. Hypertension was defined if either the systolic blood pressure (SBP) is greater than $140 \mathrm{mmHg}$ or diastolic blood pressure (DBP) is greater than $90 \mathrm{mmHg}$ or taking anti-hypertensives. Presence of diabetes was defined as either measured fasting glucose greater than $7.0 \mathrm{mmol} / \mathrm{L}$ or being diagnosed with type- 2 diabetes or taking anti-diabetic drugs or insulin. Presence of cardiovascular disease (CVD) was defined as the presence of one or more definite manifestations of coronary heart disease or stroke. Metabolic syndrome defined according to the updated National Cholesterol Education Program Adult Treatment Panel III (ATP III) criteria for Asian Americans[19,22].

To measure the presence of clinically relevant depressive symptoms, we used the Center for Epidemiologic Studies Depression Scale (CES-D) [23], which has been previously validated in Chinese populations [24,25]. Using a generally accepted cutoff point of 16 for the sum of scores, CES-D score of 0-15 was defined as no or minimal depression and $\geq 16$ was defined as minor depression $[23,26]$ to be consistent with the previous publications $[18,27,28]$.

\section{Laboratory methods}

In order to evaluate the association between SRH and cardio-metabolic disorders (e.g. cardiovascular disease, metabolic syndrome, diabetes mellitus) we measured a number of biomarkers in our population. Overnight fasting blood samples were collected in tubes containing liquid ethylenediaminetetraacetic acid (EDTA), centrifuged at $4{ }^{\circ} \mathrm{C}$, and stored at $-80^{\circ} \mathrm{C}$ until analysis. Total cholesterol, high-density lipoprotein (HDL) cholesterol, lowdensity lipoprotein (LDL) cholesterol, triglycerides (TG), and glucose were measured enzymatically on an automatic analyzer (Hitachi 7080, Japan) with reagents purchased from Wako Pure Chemical Industries (Osaka, Japan). Plasma C-reactive protein (CRP) was measured by a particle-enhanced immunoturbidimetric assay (Ultrasensitive CRP kit, Orion Diagnostica, Espoo, Finland) using microparticles coated with anti-human CRP antibodies. Retinol-binding protein 4 (RBP4) was measured by a sandwich ELISA developed in-house, utilizing affinity-chromatography purified polyclonal and monoclonal antibodies generated against recombinant human RBP4, more details can be found elsewhere [29]. Interleuikin-6 (IL-6) was measured with high-sensitivity enzyme-linked immuno-sorbent assay (ELISA) (Quantikine HS IL-6 Immunoassay, R\&D Systems, Inc., Minneapolis, Minnesota) with a low detect limit of $0.04 \mathrm{pg} / \mathrm{ml}$.

Plasma adiponectin, resistin and active plasminogen activator inhibitor-1 (PAI-1) concentrations were measured by Luminex xMAPTM Technology (Linco Research Inc, St Charles, Mo) on a Bio-Rad Multiplex Suspension Array System (more details can be found elsewhere [18]. Fasting insulin was determined by radioimmunoassay (Linco Research, MO). Index of insulin resistance was calculated using updated homeostasis model assessment methods (HOMA2-IR, using the HOMA2 calculator, http:// www.dtu.ox.ac.uk) [30].

\section{Statistical analyses}

First, we evaluated the distribution of SRH by gender, geographic region and area of residence. Then using the chisquare test, analysis of variance or Wilcoxon rank-sum test, we evaluated the associations between SRH and potential determinants, based on previous literature (Table 1). Factors that significantly modified SRH in the univariate analysis or that were consistently reported as significant determinants in previous publications were included in multivariate analysis (Table 2).

All analyses were repeated stratifying by gender, region and residence one at a time and by stratifying for two of these factors simultaneously.

The associations between SRH and biomarkers of cardiometabolic disease were tested by parametric t-test (after logarithmic transformation of the data if deemed necessary to satisfy normality assumptions), or/and non-parametric Wilcoxon rank-sum test. Biomarkers included were: resistin, PAI-1, RBP4, adiponectin, IL-6, CRP, insulin, HOMA-IR, HOMA2-IR, SBP, DBP, HDL cholesterol, LDL cholesterol, total cholesterol and triglycerides. These analyses were performed on the total population and on a sub-sample of participants that were free from CVD, cancer and diabetes.

Statistical analyses were performed with the SAS statistical package version 9.1 (SAS Institute, Cary, NC). Statistical tests were two-sided and at the 5\% level of significance.

\section{Results \\ Characteristics of the participants}

The mean age of the participants was 58.6. There were more women than men (55.7\% vs. $44.3 \%)$ (Table 3$)$. Par- 
Table I: Characteristics of the study participants by self-rated health status*

\begin{tabular}{|c|c|c|c|c|}
\hline Variable & $\begin{array}{c}\text { Total } \\
(\mathrm{n}=3287)\end{array}$ & $\begin{array}{c}\text { Good } \\
(n=105 I)\end{array}$ & $\begin{array}{l}\text { Fair/Poor } \\
(n=2236)\end{array}$ & $P$ Value \\
\hline Age, mean (SD), y & $58.6(6.0)$ & $58.00(5.99)$ & $58.89(6.00)$ & $<0.0001$ \\
\hline Age category, yrs & & & & $<0.0001$ \\
\hline $50-59$ & $1855(56.40)$ & $651(61.94)$ & $1203(53.80)$ & \\
\hline $60-70$ & $1433(43.60)$ & $400(38.06)$ & $1033(46.20)$ & \\
\hline Female gender & $|83|(55.70)$ & $530(50.43)$ & $130 \mid(58.18)$ & $<0.0001$ \\
\hline Beijing region & 1640 (49.89) & $489(46.53)$ & 1151 (5I.48) & 0.0081 \\
\hline Rural residence & $1649(50.17)$ & $672(63.94)$ & $977(43.69)$ & $<0.0001$ \\
\hline Married currently & $2880(87.62)$ & 941 (89.53) & $1939(86.72)$ & 0.0222 \\
\hline Living status (alone, $\mathrm{n}(\%)$ ) & $186(5.66)$ & $50(4.76)$ & $136(6.09)$ & 0.1245 \\
\hline Educational level, years in school & & & & $<0.0001$ \\
\hline $0-6$ & $1360(4 \mid .38)$ & $509(48.43)$ & 851 (38.06) & \\
\hline $7-9$ & $1172(35.66)$ & $354(33.68)$ & $818(36.58)$ & \\
\hline$\geq 10$ & $755(22.97)$ & $188(17.89)$ & $567(25.36)$ & \\
\hline Annual income, Yuan & & & & 0.0015 \\
\hline$<10000$ & $908(29.04)$ & $335(33.27)$ & $573(27.03)$ & \\
\hline $10000-29999$ & $1422(45.47)$ & $434(43.10)$ & $988(46.60)$ & \\
\hline$\geq 30000$ & $797(25.49)$ & $238(23.63)$ & $559(26.37)$ & \\
\hline Employment & & & & $<0.0001$ \\
\hline employed & $784(23.85)$ & $336(31.97)$ & $448(20.04)$ & \\
\hline retired & $1819(55.34)$ & $465(44.24)$ & $1354(60.55)$ & \\
\hline unemployed/on welfare & $684(20.81)$ & $250(23.79)$ & $434(19.41)$ & \\
\hline Social activities (YES, n(\%)) & $1670(50.81)$ & $54 \mid(5 I .47)$ & $1129(50.49)$ & 0.5991 \\
\hline Smoking & & & & $<0.0001$ \\
\hline current & $918(27.93)$ & $362(34.44)$ & $556(24.87)$ & \\
\hline former & $329(10.01)$ & $94(8.94)$ & $235(10.5 \mathrm{I})$ & \\
\hline never & $2040(62.06)$ & $595(56.61)$ & I 445 (64.62) & \\
\hline Current alcohol drinker & $938(28.54)$ & $349(33.21)$ & $589(26.34)$ & $<0.0001$ \\
\hline Physical activity, level & & & & $<0.0001$ \\
\hline low & $245(7.45)$ & $63(5.99)$ & $182(8.14)$ & \\
\hline moderate & $1379(4 \mid .95)$ & $343(32.64)$ & $1036(46.33)$ & \\
\hline high & 1663 (50.59) & $645(61.37)$ & $1018(45.53)$ & \\
\hline Sleep quality & & & & $<0.0001$ \\
\hline well & $1745(53.20)$ & $76 \mid(72.55)$ & $984(44.11)$ & \\
\hline common & $1002(30.55)$ & $204(19.45)$ & $798(35.77)$ & \\
\hline poor & $533(16.25)$ & $84(8.01)$ & $449(20.13)$ & \\
\hline Sleep quantity, total hours of sleep/day & & & & $<0.0001$ \\
\hline$<7$ & $809(24.66)$ & $201(19.22)$ & $608(27.22)$ & \\
\hline $7-9$ & $2211(67.41)$ & 769 (73.52) & $1442(64.55)$ & \\
\hline$>9$ & $260(7.93)$ & $76(7.27)$ & $184(8.24)$ & \\
\hline Medical insurance (YES, $n(\%))$ & $2297(70.09)$ & $693(66.13)$ & $1604(71.96)$ & 0.0007 \\
\hline Waist circumference $(\mathrm{cm})$, mean (SD) & $83.74(10.55)$ & $82.89(10.06)$ & $84.13(10.75)$ & 0.0017 \\
\hline Hip circumference(cm), mean (SD) & $93.61(6.85)$ & $92.91(6.48)$ & $93.94(7.00)$ & $<0.0001$ \\
\hline BMI, mean (SD) & $24.46(3.59)$ & $24.20(3.39)$ & $24.58(3.67)$ & 0.0053 \\
\hline BMI category & & & & 0.0061 \\
\hline under weight & III (3.38) & $37(3.52)$ & $74(3.31)$ & \\
\hline normal & 1423 (43.29) & $495(47.10)$ & $928(41.50)$ & \\
\hline over weight & $1266(38.52)$ & $389(37.01)$ & $877(39.22)$ & \\
\hline obese & $487(14.82)$ & $130(12.37)$ & $357(15.97)$ & \\
\hline Hypertension (YES, n(\%)) & $1794(54.58)$ & $493(46.91)$ & $1301(58.18)$ & $<.0001$ \\
\hline Diabetes (YES, $n(\%)$ ) & $446(13.95)$ & $79(7.68)$ & $367(16.92)$ & $<0.0001$ \\
\hline CVD (YES, n(\%)) & $333(10.36)$ & $27(2.61)$ & $306(14.04)$ & $<0.0001$ \\
\hline Metabolic syndrome (YES, n(\%)) & $|40|(42.62)$ & $352(33.49)$ & $1049(46.91)$ & $<0.0001$ \\
\hline Crude CES-D scores, mean (SD) & $5.07(7.88)$ & $2.89(5.36)$ & $6.09(8.62)$ & $<0.0001$ \\
\hline Depressive Symptoms (YES, n(\%)) & $311(9.46)$ & $38(3.62)$ & $273(12.21)$ & $<0.0001$ \\
\hline
\end{tabular}

Abbreviations: SD, standard deviation; CES-D, Center for Epidemiologic Studies Depression Scale; BMI, Body Mass Index; CVD, Cardio-Vascular Disease; $\mathrm{Cl}$, Confidence interval.

*Data are presented as frequency (percentage) unless otherwise indicated.

There are two missing values for SRH and therefore the total number of 3287 subjects were included in the analysis. 
Table 2: Multivariate adjusted determinants of self-rated health status (good/poor) for the total population and stratified population by residential location or gender

\begin{tabular}{|c|c|c|c|c|c|}
\hline & \multicolumn{5}{|c|}{ Association with self rated health status (OR with $95 \% \mathrm{Cl}$ ) } \\
\hline & $\begin{array}{l}\text { Total } \\
(n=3289)\end{array}$ & $\begin{array}{l}\text { Rural } \\
(\mathrm{n}=1649)\end{array}$ & $\begin{array}{l}\text { Urban } \\
(n=1640)\end{array}$ & $\begin{array}{l}\text { Female } \\
(n=1831)\end{array}$ & $\begin{array}{l}\text { Male } \\
(n=1458)\end{array}$ \\
\hline $\begin{array}{l}\text { Gender } \\
\text { (female vs. male) }\end{array}$ & $1.03(0.79-1.33)$ & $1.38(0.96-2.00)$ & $0.84(0.57-1.23)$ & & \\
\hline $\begin{array}{l}\text { Residential location } \\
\text { (rural vs. urban) }\end{array}$ & $1.89(1.47-2.43)^{*}$ & & & $1.81(1.25-2.63)^{*}$ & $2.00(1.40-2.86)^{*}$ \\
\hline $\begin{array}{l}\text { Geographic location } \\
\text { (Beijing vs. Shanghai) }\end{array}$ & I.II (0.92-I.33) & $0.77(0.59-1.02)$ & $1.45(1.10-1.91)^{*}$ & $0.92(0.7|-| .2 I)$ & $1.22(0.93-1.59)$ \\
\hline $\begin{array}{l}\text { Age group } \ddagger \\
\text { (group I vs. } 2 \text { ) }\end{array}$ & $1.08(0.89-1.32)$ & $1.29(0.99-1.68)$ & $0.85(0.62-1.18)$ & $1.20(0.92-1.57)$ & $1.07(0.78-1.48)$ \\
\hline $\begin{array}{l}\text { Marital status } \\
\text { (spouse vs. no spouse) }\end{array}$ & I.II (0.84-I.46) & $1.20(0.83-1.72)$ & $0.91(0.59-1.42)$ & $1.30(0.91-1.85)$ & $0.84(0.53-1.33)$ \\
\hline Educational level, years in school $\dagger$ & & & & $*$ & \\
\hline group I vs. 3 & $1.21(0.90-1.63)$ & $0.76(0.37-1.56)$ & $1.17(0.75-1.83)$ & $1.87(1.20-2.90)^{*}$ & $0.83(0.53-1.28)$ \\
\hline group 2 vs. 3 & $1.03(0.80-1.33)$ & $0.73(0.36-1.48)$ & $1.14(0.85-1.53)$ & $1.56(1.06-2.31)^{*}$ & $0.76(0.53-1.10)$ \\
\hline \multicolumn{6}{|l|}{ Annual income } \\
\hline high vs. low & $1.38(1.05-1.82)^{*}$ & $1.40(0.94-2.09)$ & $1.23(0.70-2.15)$ & $1.32(0.91-1.93)$ & $1.39(0.92-2.09)$ \\
\hline medium vs. low & $1.17(0.94-1.46)$ & $1.18(0.92-1.52)$ & $1.02(0.60-1.76)$ & I.II (0.82-I.50) & $1.16(0.84-1.60)$ \\
\hline Employment & * & & & & \\
\hline employed vs. retired & $1.36(\mid .08-1.71)^{*}$ & $1.37(1.01-1.85)^{*}$ & $1.37(0.94-1.99)$ & $1.36(0.96-1.93)$ & $1.35(0.96-1.90)$ \\
\hline unemployed vs. retired & $1.26(0.99-1.61)$ & $1.29(0.96-1.74)$ & $0.86(0.46-1.58)$ & $1.32(0.94-1.85)$ & $1.13(0.76-1.69)$ \\
\hline Smoking & & & & * & \\
\hline current vs. never smoker & $1.16(0.89-1.5 \mathrm{I})$ & $1.17(0.81-1.68)$ & $1.33(0.90-1.96)$ & $1.85(1.11-3.10)^{*}$ & $1.00(0.73-1.37)$ \\
\hline former vs. never smoker & $0.90(0.63-1.27)$ & $0.96(0.60-1.54)$ & $0.96(0.55-1.66)$ & $1.59(0.69-3.66)$ & $0.77(0.52-1.15)$ \\
\hline $\begin{array}{l}\text { Alcohol drinking } \\
\text { (yes vs. no) }\end{array}$ & $1.26(1.02-1.57)^{*}$ & $1.64(1.21-2.23)^{*}$ & $\mathrm{I} .03(0.75-\mathrm{I} .4 \mathrm{I})$ & $1.10(0.73-1.66)$ & $1.35(1.04-1.75)^{*}$ \\
\hline Physical activity & $*$ & & $*$ & $*$ & $*$ \\
\hline high vs. low & $1.64(1.16-2.33)^{*}$ & $1.56(1.03-2.36)^{*}$ & $1.94(0.99-3.79)$ & $1.63(1.02-2.59)^{*}$ & $1.76(1.03-3.01)^{*}$ \\
\hline moderate vs. low & $1.23(0.86-1.78)$ & $1.59(1.01-2.5 \mathrm{I})^{*}$ & $1.14(0.58-2.22)$ & $1.26(0.78-2.05)$ & $1.29(0.73-2.26)$ \\
\hline Sleep quality & $*$ & $*$ & $*$ & * & * \\
\hline well vs. poor & $2.95(2.22-3.91)^{*}$ & $3.52(2.4 \mathrm{I}-5.16)^{*}$ & $2.26(1.49-3.45)^{*}$ & $3.10(2.17-4.44)^{*}$ & $2.77(1.73-4.43)^{*}$ \\
\hline common vs. poor & $1.11(0.82-1.50)$ & $1.27(0.84-1.93)$ & $0.90(0.57-\mid .4 I)$ & $1.12(0.76-1.65)$ & $1.08(0.65-1.80)$ \\
\hline Hypertension (no vs. yes) & $1.39(1.17-1.66)^{*}$ & $1.37(1.08-1.73)^{*}$ & $1.39(1.07-1.82)^{*}$ & $1.23(0.96-1.56)$ & $1.63(1.27-2.09)^{*}$ \\
\hline Diabetes (no vs. yes) & $2.03(1.53-2.69)^{*}$ & $1.65(1.12-2.42)^{*}$ & $2.87(1.85-4.43)^{*}$ & $1.76(1.17-2.65)^{*}$ & $2.36(1.59-3.50)^{*}$ \\
\hline CVD (no vs. yes) & $3.68(2.39-5.66)^{*}$ & $5.87(2.85-12.09)^{*}$ & $2.80(1.63-4.81)^{*}$ & $2.92(1.62-5.27)^{*}$ & $4.94(2.57-9.48)^{*}$ \\
\hline $\begin{array}{l}\text { Depressive Symptoms } \\
\text { (no vs. yes) }\end{array}$ & $2.50(1.67-3.73)^{*}$ & $2.71(1.64-4.49)^{*}$ & $1.93(1.01-3.71)^{*}$ & $3.26(1.90-5.59)^{*}$ & $1.70(0.90-3.18)$ \\
\hline
\end{tabular}

Abbreviations: CES-D, Center for Epidemiologic Studies Depression Scale; CVD, Cardio-Vascular Disease; OR, Odds ratio; Cl, Confidence interval. * Statistically significant at a P-value level below 0.05 .

† Group I, 2 and 3 for educational level are 0-6, 7-9 and $\geq 10$ years in school respectively.

$\ddagger$ Group I, 2 for age are $50-<60$ and $60-70$ years old respectively.

ticipants were mostly married $(88 \%)$, more than half of the participants were retired, 51\% reported to be actively involved in social activities and 53\% to have a good sleep quality. Although the majority of the participants were non-smokers $(72.1 \%$ total, $95 \%$ women and $44 \%$ men), reported not to drink alcohol $(71.4 \%$ total, $90 \%$ women and $48 \%$ men) and had moderate or high levels of physical activity (92.5\% total, 92\% women and 93\% men), more than half of the population were overweight or obese ( $53.4 \%$ total, $56 \%$ women, $50 \%$ men). Nine percent of participants had depressive symptoms, $14 \%$ diabetes, $10 \%$ CVD, $55 \%$ hypertension and $43 \%$ had metabolic syndrome (Table 3 ).

\section{Distribution of SRH}

One third of the study population reported their health status as good or very good (32\%), almost half of the population reported their health as fair (53\%) and the rest as poor or very poor $(15 \%)$ (Table 3 ).

Sixty-four percent of those that reported good SRH were rural residents. Those with poor SRH were more likely to be retired compared to those with good SRH (60.5\% vs. $44.2 \%)$. Lower prevalence of high physical activity $(45.5 \%$ vs. $61.4 \%)$ and good sleep quality $(44.1 \%$ vs. $72.5 \%)$ and higher prevalence of CVD (14\% vs. $2.6 \%)$, diabetes $(16.9 \%$ vs. $7.7 \%)$ and depressive symptoms 
Table 3: Characteristics of the study participants by residential location*

\begin{tabular}{|c|c|c|c|c|}
\hline Variable & $\begin{array}{c}\text { Total } \\
(n=3289)\end{array}$ & $\begin{array}{c}\text { Rural } \\
(n=\mid 649)\end{array}$ & $\begin{array}{c}\text { Urban } \\
(n=1640)\end{array}$ & P Value \\
\hline Self-rated health status & & & & $<0.0001$ \\
\hline excellent and good & $105 \mid(31.97)$ & $672(40.75)$ & $379(23.14)$ & \\
\hline fair & $1733(52.72)$ & $700(42.45)$ & $1033(63.06)$ & \\
\hline poor and very poor & $503(15.30)$ & $277(16.80)$ & $226(13.80)$ & \\
\hline Age, mean (SD) & $58.6(6.0)$ & $58.4(5.8)$ & $58.8(6.2)$ & 0.01 \\
\hline \multicolumn{5}{|l|}{ Age category, yrs } \\
\hline $50-59$ & $1855(56.40)$ & $958(58.10)$ & $897(54.70)$ & 0.0492 \\
\hline $60-70$ & $1434(43.60)$ & $691(41.90)$ & $743(45.30)$ & \\
\hline Female gender & $183 \mid(55.67)$ & $906(54.94)$ & $925(56.40)$ & 0.3994 \\
\hline Beijing region & I64I (49.89) & $812(49.24)$ & $829(50.55)$ & 0.4536 \\
\hline Married currently & $2882(87.63)$ & $1436(87.08)$ & $1446(88.17)$ & 0.3436 \\
\hline Living status (alone, n (\%)) & $186(5.66)$ & $104(6.31)$ & $82(5.00)$ & 0.1056 \\
\hline Educational level, years in school & & & & $<.0001$ \\
\hline $0-6$ & $1360(4 \mid .35)$ & $1107(67.13)$ & $253(15.43)$ & \\
\hline $7-9$ & $1172(35.63)$ & $497(30.14)$ & $675(41.16)$ & \\
\hline$\geq 10$ & $757(23.02)$ & $45(2.73)$ & $712(43.4 I)$ & \\
\hline Annual income, Yuan & & & & $<.0001$ \\
\hline$<10000$ & $909(29.05)$ & $790(50.74)$ & 119 (7.57) & \\
\hline $10000-29999$ & $1422(45.45)$ & $613(39.37)$ & $809(51.46)$ & \\
\hline$\geq 30000$ & $798(25.50)$ & $154(9.89)$ & $644(40.97)$ & \\
\hline Employment & & & & $<0.0001$ \\
\hline employed & $785(23.87)$ & $517(31.35)$ & $268(16.34)$ & \\
\hline retired & $1819(55.31)$ & $556(33.72)$ & $1263(77.01)$ & \\
\hline unemployed/on welfare & $685(20.83)$ & $576(34.93)$ & $109(6.65)$ & \\
\hline Social activities (Yes, n (\%)) & $167 \mid(50.81)$ & $626(37.96)$ & $1045(63.72)$ & $<0.0001$ \\
\hline Smoking & & & & $<.0001$ \\
\hline current & $919(27.94)$ & $513(31.11)$ & $406(24.76)$ & \\
\hline former & $329(10.00)$ & $180(10.92)$ & $149(9.09)$ & \\
\hline never & $2041(62.06)$ & $956(57.97)$ & $1085(66.16)$ & \\
\hline Current alcohol drinker & $940(28.58)$ & $443(26.86)$ & $497(30.30)$ & 0.03 \\
\hline Physical activity, level & & & & $<.0001$ \\
\hline low & $245(7.45)$ & 161 (9.76) & $84(5.12)$ & \\
\hline moderate & $|38|(4 \mid .99)$ & $400(24.26)$ & $981(59.82)$ & \\
\hline high & $1663(50.56)$ & $1088(65.98)$ & $575(35.06)$ & \\
\hline Sleep quality & & & & $<0.0001$ \\
\hline well & $1746(53.20)$ & $959(58.26)$ & $787(48.11)$ & \\
\hline common & $1003(30.56)$ & $422(25.64)$ & $581(35.51)$ & \\
\hline poor & $533(16.24)$ & $265(16.10)$ & $268(16.38)$ & \\
\hline Sleep quantity, total hours of sleep/day & & & & $<0.0001$ \\
\hline$<7$ & $810(24.68)$ & $390(23.68)$ & $420(25.69)$ & \\
\hline $7-9$ & $2212(67.40)$ & $1089(66.12)$ & $1123(68.69)$ & \\
\hline$>9$ & $260(7.92)$ & $168(10.20)$ & $92(5.63)$ & \\
\hline Waist circumference(cm), mean (SD) & $83.74(10.55)$ & $82.61(10.73)$ & $84.87(10.24)$ & $<0.0001$ \\
\hline Hip circumference $(\mathrm{cm})$, mean (SD) & $93.61(6.85)$ & $91.91(6.40)$ & $95.32(6.86)$ & $<0.0001$ \\
\hline BMI, mean (SD) & 24.46 (3.59) & $24.05(3.62)$ & $24.88(3.5 I)$ & $<0.0001$ \\
\hline BMI category & & & & $<0.0001$ \\
\hline under weight & III (3.37) & 74 (4.49) & $37(2.26)$ & \\
\hline normal & 1423 (43.27) & $788(47.79)$ & $635(38.72)$ & \\
\hline over weight & $1268(38.55)$ & $570(34.57)$ & $698(42.56)$ & \\
\hline obese & $487(|4.8|)$ & $217(13.16)$ & $270(16.46)$ & \\
\hline Medical insurance (Yes, n (\%)) & $2299(70.11)$ & $768(46.69)$ & $|53|(93.70)$ & $<.0001$ \\
\hline Hypertension (Yes, n (\%)) & $1796(54.61)$ & $930(56.40)$ & $866(52.80)$ & 0.0385 \\
\hline Diabetic (Yes, n (\%)) & 447 (13.97) & $184(11.37)$ & $263(16.64)$ & $<0.0001$ \\
\hline CVD (Yes, n (\%)) & $333(10.35)$ & $125(7.69)$ & $208(13.07)$ & $<0.0001$ \\
\hline Metabolic syndrome (Yes, n (\%)) & 1402 (42.63) & $625(37.90)$ & 777 (47.38) & $<0.0001$ \\
\hline Crude CES-D scores, mean (SD) & $5.07(7.88)$ & $5.27(8.4 I)$ & $4.87(7.30)$ & 0.40 \\
\hline Depressive Symptoms (Yes, n (\%)) & $312(9.49)$ & $169(10.25)$ & 143 (8.72) & 0.1346 \\
\hline
\end{tabular}


$(12.2 \%$ vs. $3.6 \%)$ was observed among those that reported poor SRH compared to their counterparts (Table $1)$.

\section{Associated factors with Self-Rated Health}

Based on univariate analysis (results not shown, only pvalues presented in the Table 1), males, younger people (50-60 yrs), rural residents and those living in Shanghai were more likely to have a good SRH. Single (without spouse) individuals, retired and those with higher level of education or annual income are less likely to report a good SRH. Physically active people, smokers and drinkers had higher odds to report a good level of SRH. Also benefiting from a good sleep quality was significantly associated with a good level of SRH. Depressed, CVD and diabetic participants and those with hypertension and metabolic syndrome were more likely to have a poor SRH. Social activity levels and living status showed no association with SRH in the univariate analysis and were therefore not included in the multivariate analyses.

After adjusting for all selected factors in the multivariate analysis, living in rural area (Odds Ratio (OR) 1.89; 95\% Confidence Interval (CI)1.47-2.43), being employed (OR 1.36; 95\% CI 1.08-1.71), drinking alcohol (OR 1.26; 95\% CI 1.02-1.57), having high levels of physical activity (OR 1.64; 95\% CI 1.16-2.33) and benefiting from good sleep quality (OR 2.95; 95\% CI 2.22-3.91) increased the chance of reporting a good SRH. Furthermore, being free of diabetes (OR 2.03; 95\% CI 1.53-2.69), hypertension (OR1.39; 95\% CI 1.17-1.66), CVD (OR 3.68; 95\% CI 2.39-5.66) and depression (OR 2.50; 95\% CI 1.67-3.73) independent of the other determinants significantly increased the chance of feeling healthier (Table 2).

Similar findings were obtained when the analyses were stratified by residence (urban vs. rural) or gender. Quality of sleep, diabetes and CVD were found significantly associated with SRH for all subgroups. We have also found significant association of depression (apart from male), hypertension (apart from female) and physical activity (apart from rural resident) in most of the subgroups. Among rural residents, drinking alcohol (OR 1.64; 95\% CI 1.21-2.23) and among urban residents region of living (Beijing vs. Shanghai OR 1.45; CI 1.10-1.91) was significantly associated with SRH (Table 2). Educational levels and smoking (current vs. never OR 1.85; CI 1.11-3.10), would affect women's SRH while alcohol drinking (No vs. yes OR 0.74; CI 0.57-0.96) was associated with men's SRH (Table 2). Residence of living was found significant for both gender subgroups (female (rural vs. urban OR 1.81; CI 1.25-2.63), male (rural vs. urban OR 2.00; CI 1.402.86).

After stratifying the analyses by gender and residence of living simultaneously (Table 4), quality of sleep and CVD remained the most significant determinants of SRH for all subgroups. Hypertension affected males in both urban \& rural areas but not females. Region of living and diabetes were significantly associated with SRH in rural women and urban men. Depressive symptoms were significantly associated with SRH for all subgroups except urban males. Educational level, employment status and level of physical activity were only associated with urban female's SRH. Age was significant between urban males and smoking between rural males.

\section{SRH and cardio-metabolic biomarkers}

Significant differences in level of biomarkers (except for adiponectin) were observed between the participants with different level of SRH. The level of SRH inversely related to the levels of resistin, CRP, insulin, HOMA-IR, HOMA2IR, systolic and diastolic blood pressure (Table 5). Trends and significant differences remained for resistin, CRP, insulin and HOMA2-IR among a healthier subgroup of participants that was free from cancer, CVD and diabetes (data not shown).

\section{Discussion}

We found a relatively low level of good SRH among middle-aged and elderly Chinese (32\%), which varied substantially by area of residence, lifestyle and socioeconomic factors and presence of chronic diseases. Lower levels of SRH were associated with worsened levels of inflammatory markers, blood lipids, insulin and insulin resistance in both the total population and a sub sample free from CVD, diabetes and cancer.

Only one third of the elderly Chinese assessed their health as good or very good. Despite lower socio-economic status (lower average income, fewer years in full-time education and less medical insurance coverage), rural residents were almost twice as likely to feel healthier compared to urban residents. This could be the consequence of different lifestyle followed by those living in rural areas, characterized by higher levels of physical activity, lower BMI and a higher age of retirement. Furthermore, the prevalence of both CVD (7.7\% rural vs. $13 \%$ urban) and diabetes (11.4\% rural vs. $17 \%$ urban) was lower among rural residents (Table 3).

Compared to the previous studies, we found relatively lower level of good/excellent SRH. This could be due to differences in the selection criteria (age range, disease status, functional status, cognitive status, exclusion criteria, etc.) or the coverage of our sample.

In previous studies, Yu et al. [10] by studying 3094 elderly (over 65 yrs) Chinese living in urban Shanghai found a level of good/excellent SRH of approximately 49\% (15\% higher than our finding in the urban-shanghai). This difference could be due to the selection criteria used in the 
Table 4: Multivariate adjusted determinants of self-rated health status (good/poor) stratified by residential location and gender (simultaneously)

\begin{tabular}{|c|c|c|c|c|}
\hline & \multicolumn{4}{|c|}{ Association with self rated health status (OR with $95 \% \mathrm{Cl}$ ) } \\
\hline & $\begin{array}{l}\text { Rural-female } \\
(\mathrm{n}=925)\end{array}$ & $\begin{array}{l}\text { Rural-male } \\
(n=7 \mid 5)\end{array}$ & $\begin{array}{l}\text { Urban-Female } \\
(n=906)\end{array}$ & $\begin{array}{l}\text { Urban-Male } \\
(n=743)\end{array}$ \\
\hline $\begin{array}{l}\text { Geographic location } \\
\text { (Beijing vs. Shanghai) }\end{array}$ & $0.59(0.39-0.89)^{*}$ & $0.92(0.62-1.37)$ & $1.23(0.83-1.81)$ & $1.74(1.16-2.63)^{*}$ \\
\hline $\begin{array}{l}\text { Age group } \ddagger \\
\text { (group I vs. 2) }\end{array}$ & $1.22(0.85-1.75)$ & $1.49(0.98-2.28)$ & $1.29(0.83-2.02)$ & $0.55(0.32-0.95)^{*}$ \\
\hline $\begin{array}{l}\text { Marital status } \\
\text { (spouse vs. no spouse) }\end{array}$ & $1.49(0.92-2.42)$ & $0.78(0.43-1.42)$ & $0.97(0.55-1.70)$ & $0.90(0.42-1.94)$ \\
\hline Educational level, years in school $\dagger$ & & & * & \\
\hline group I vs. 3 & $1.25(0.31-5.08)$ & $0.62(0.25-1.56)$ & $2.27(1.24-4.17)^{*}$ & $0.53(0.24-1.18)$ \\
\hline group 2 vs. 3 & $1.37(0.34-5.63)$ & $0.51(0.21-1.26)$ & $1.52(0.99-2.35)$ & $0.95(0.61-1.47)$ \\
\hline \multicolumn{5}{|l|}{ Annual income } \\
\hline high vs. low & $1.43(0.82-2.49)$ & $1.34(0.74-2.42)$ & $1.12(0.53-2.37)$ & $1.37(0.57-3.27)$ \\
\hline medium vs. low & $1.23(0.87-1.74)$ & $1.11(0.76-1.63)$ & $0.93(0.45-1.93)$ & I.II (0.48-2.56) \\
\hline Employment & & & * & \\
\hline employed vs. retired & $1.10(0.7 \mid-1.70)$ & $1.59(0.99-2.55)$ & $2.26(1.25-4.08)^{*}$ & $1.36(0.79-2.35)$ \\
\hline unemployed vs. retired & $1.21(0.83-1.77)$ & $1.36(0.81-2.28)$ & $0.55(0.11-2.65)$ & $1.08(0.50-2.33)$ \\
\hline Smoking & $*$ & & & \\
\hline current vs. never smoker & $2.53(1.27-5.04)^{*}$ & $0.80(0.5|-| .26)$ & $1.45(0.61-3.43)$ & $1.37(0.87-2.17)$ \\
\hline former vs. never smoker & $1.52(0.50-4.66)$ & $0.72(0.4 I-1.25)$ & $2.17(0.58-8.12)$ & $0.87(0.47-1.62)$ \\
\hline $\begin{array}{l}\text { Alcohol drinking } \\
\text { (yes vs. no) }\end{array}$ & $1.15(0.59-2.27)$ & $1.76(1.23-2.52)^{*}$ & $1.08(0.63-1.85)$ & $1.06(0.70-1.58)$ \\
\hline Physical activity & & & $*$ & \\
\hline high vs. low & $1.39(0.81-2.39)$ & $2.01(1.03-3.93)^{*}$ & $2.42(0.90-6.52)$ & $1.76(0.67-4.59)$ \\
\hline moderate vs. low & $1.75(0.97-3.17)$ & $1.62(0.77-3.39)$ & $1.22(0.46-3.25)$ & $1.14(0.44-2.99)$ \\
\hline Sleep quality & * & * & * & * \\
\hline well vs. poor & $4.09(2.54-6.58)^{*}$ & $2.53(1.30-4.91)^{*}$ & $1.95(1.14-3.34)^{*}$ & $3.04(1.50-6.13)^{*}$ \\
\hline common vs. poor & $1.49(0.89-2.49)$ & $0.95(0.45-1.98)$ & $0.73(0.40-1.33)$ & $1.16(0.55-2.42)$ \\
\hline Diabetic (no vs. yes) & $1.83(1.04-3.21)^{*}$ & $1.54(0.88-2.67)$ & $1.77(0.95-3.29)$ & $4.35(2.30-8.22) *$ \\
\hline Hypertension (no vs. yes) & $1.14(0.82-1.58)$ & $1.74(1.23-2.45)^{*}$ & $1.27(0.87-1.86)$ & $1.48(1.00-2.18)^{*}$ \\
\hline CVD (no vs. yes) & $3.55(1.44-8.76)^{*}$ & $13.34(3.11-57.28)^{*}$ & $2.51(1.13-5.56)^{*}$ & $3.32(1.56-7.05)^{*}$ \\
\hline $\begin{array}{l}\text { Depressive Symptoms } \\
\text { (no vs. yes) }\end{array}$ & $2.69(1.42-5.09)^{*}$ & $2.82(1.17-6.83)^{*}$ & $4.14(1.41-12.10)^{*}$ & $0.87(0.35-2.16)$ \\
\hline
\end{tabular}

Abbreviations: CES-D, Center for Epidemiologic Studies Depression Scale; CVD, Cardio-Vascular Disease; OR, Odds ratio; Cl, Confidence interval.

* Statistically significant at a P-value level below 0.05 .

† Group I, 2 and 3 for educational level are 0-6, 7-9 and $\geq 10$ years in school respectively.

$\ddagger$ Group I, 2 for age are $50-<60$ and $60-70$ years old respectively.

study. Moreover, Lim et al. [2] studied 6236 individuals aged 18 and above (76\% under 50 yrs) in Singapore (urban area) and found a level of good/excellent SRH of $77 \%$ (54\% higher than our findings for urban residents) and Pei et al. [13] by studying 9594 aged 18 and over Chinese, found a level of good SRH of $72 \%$. The difference between our study and these two studies could be due to a high proportion of study participants below age 50 and therefore a lower prevalence of comorbidities and other factors that increase with age and significantly modify SRH. Consistent with our findings, Pei et al. [13] found a significant association between residential affiliation and $\mathrm{SRH}$, suggesting that living in rural area decreases the chance of reporting poor SRH.

Similar to our findings, Leung et al. [8] by studying 411 elderly ( 65 and over) Chinese living in two long-term care institutions found a relatively lower level of good/excellent SRH of approximately $33 \%$. This could be due to their selection criteria (retired government employees, single or widows or poor or homeless participants who needed care (55\% suffered from 4 or more chronic illnesses)) and older age (65\% over 75 years). Goldman et al. [15] also showed the level of good SRH of approximately $25 \%$ in elderly Taiwan residents which could be due to the older sample population. Different levels of good SRH have also been reported for other countries such as USA [16] $(66 \%)$, and Norway [17] (65\%).

We found a significant association between different lifestyle characteristics and SRH in the current analyses. High levels of physical activity were associated with better SRH. Sleep quality was one of the factors which had the highest level of association with SRH in the study population. 
Table 5: Cardio-metabolic risk factors and biomarkers of the study participants by self rated health status

\begin{tabular}{|c|c|c|c|c|c|}
\hline Variable, mean (SD) & $\begin{array}{c}\text { Total } \\
(n=3289)\end{array}$ & $\begin{array}{c}\text { Good } \\
(n=1051)\end{array}$ & $\begin{array}{c}\text { Fair } \\
(n=1733)\end{array}$ & $\begin{array}{c}\text { Poor } \\
(n=503)\end{array}$ & $P$ Value\# \\
\hline Resistin & $11.45(9.36)$ & $11.03(9.44)$ & $11.42(8.62)$ & $12.49(11.37)$ & $0.01208^{*}$ \\
\hline PAI-I & $14.72(18.13)$ & $|3.3|(\mid 7.45)$ & $15.80(\mid 8.67)$ & $13.83(\mid 7.21)$ & $<0.000 I^{*}$ \\
\hline RBP4 & $40.1(11.75)$ & $39.05(11.39)$ & $40.74(11.84)$ & $40.08(12.05)$ & $0.0014^{*}$ \\
\hline Adipo & $16.50(11.65)$ & $16.06(11.49)$ & $16.50(\mid 1.51)$ & $17.4 \mid(12.38)$ & 0.252 \\
\hline IL-6 & $1.55(2.77)$ & $\mathrm{I} .49(2.52)$ & $\mathrm{I} .45(2.29)$ & $2.02(4.30)$ & $0.0003 *$ \\
\hline CRP & $1.57(4.29)$ & $1.23(2.12)$ & $1.66(5.22)$ & $1.97(4.06)$ & $<0.0001 *$ \\
\hline Insulin & I5.44(9.48) & $14.27(9.13)$ & $15.67(8.40)$ & $17.12(12.83)$ & $<0.0001^{*}$ \\
\hline Homa-ir & $4.09(3.35)$ & $3.61(2.66)$ & $4.18(3.08)$ & $4.78(5.03)$ & $<0.0001^{*}$ \\
\hline Homa2-ir & $0.30(0.22)$ & $0.28(0.26)$ & $0.31(0.17)$ & $0.34(0.27)$ & $<0.0001^{*}$ \\
\hline SBP & $140.12(22.47)$ & $138.62(22.13)$ & $139.72(22.36)$ & $144.66(23.04)$ & $<0.0001 *$ \\
\hline DBP & $80.17(10.80)$ & $79.69(10.38)$ & $80.05(11.02)$ & $81.56(10.83)^{\prime}$ & $<0.005^{*}$ \\
\hline HDL & $1.28(0.33)$ & $\mathrm{I} .3 \mathrm{I}(0.34)$ & $1.26(0.33)$ & $1.27(0.34)$ & $<0.0018^{*}$ \\
\hline LDL & $3.26(0.97)$ & $3.14(0.94)$ & $3.33(0.97)$ & $3.28(1.00)$ & $<0.000 I^{*}$ \\
\hline $\mathrm{TCH}$ & $4.70(0.98)$ & $4.58(0.94)$ & $4.76(0.98)$ & $4.7 I(I .03)$ & $<0.000 I^{*}$ \\
\hline TG & $1.39(1.07)$ & $1.24(0.92)$ & $1.48(1.17)$ & $1.39(0.96)$ & $<0.000 I^{*}$ \\
\hline
\end{tabular}

Abbreviations: PAI_I, plasminogen activator inhibitor-I; RBP4, retinol binding protein 4; ADIPO, adiponectin; IL6, interleukin-6; IL6, interleukin-6; CRP, c-reactive protein; Homa-ir, homeostasis model assessment of insulin resistance (fasting glucose $(\mathrm{mmol} / \mathrm{L}) \times$ fasting insulin $(\mu \mathrm{U} / \mathrm{ml}) / 22.5) ; \mathrm{SBP}$, systolic blood pressure; DBP, diastolic blood pressure; HDL, high-density lipoprotein; LDL, low-density lipoprotein; TCH, total cholesterol; TG, triglycerides; SD, standard deviation;

\# Wilcoxon/t-test $\mathrm{p}$-value that indicates the difference of the levels of biomarkers within the levels of SRH; $* \mathrm{P}<0.05$

Those with good sleep quality were almost twice likely to report good levels of SRH. This result was consistent for all subgroups (regional, residential and gender) of the study population. The effect of smoking and alcohol drinking on SRH could be hampered by the effect of gender as the majority of Chinese smokers and drinkers were men. Nevertheless, we found a significant association of alcohol intake and SRH on the total population and among rural residents. The association however indicated the higher chance of reporting good SRH for those drinking alcohol. This could be due to the lack of information about drinking habits (number of units or frequency of alcohol consumption) in the models.

Furthermore, socio-economic factors, such as education and employment status, affected mainly urban women. Most of the previous studies $[12,13,31]$ indicated that lower levels of income or education were significantly associated with reporting poor SRH. Consistent with previous publications $[12,31]$, we found a significant difference in the level of SRH of those reported high and low level of income in the total population but no association of income in any other subgroups of the study population. A significant association of education has only been found in females and urban females subgroups. This could be due to the fact that the area of residence might be a more comprehensive factor in our study population and might partially cover the effect of socio-economic factors (e.g. the Chinese population in our sample is skewed towards lower income and education and late retirement in rural areas).
Absence of CVD, hypertension, diabetes or depression increased the chance of reporting a good level of SRH. Presence of depressive symptoms had a bigger impact on women and urban residents. Suffering from CVD was found consistently associated with SRH levels for all subgroups of gender and area of residence with the strongest association among rural-men as more likely to report poor SRH. The deleterious association of CVD presence on SRH was higher among rural residents and men compared to urban residents and women. Consistent with our findings, by studying 1589 elderly Chinese in Hong Kong, Cheng et al. [11] found a strong association between SRH and chronic illnesses and sleep quality. Also Li et al. [1] completed a study on 56162 Chinese elderly in Hong Kong and found a significant association between time comparative SRH and active chronic disease and depression. Lim et al. [2] by studying 6236 individuals aged 18 and above in Singapore found a significant association between SRH and reported illnesses (cancer and CVD) and income.

Lower concentrations of inflammatory biomarkers, plasma insulin and index of insulin resistance were associated with good levels of SRH for the total population and among a subgroup of participants that were free from cancer, CVD and diabetes. Levels of SRH might effectively reveal early deviations from healthy trajectories, making SRH an effective assessment tool to evaluate healthy aging targeting the early prevention of the appearance of chronic disease. To our knowledge, no study has been conducted on Chinese in mainland China to examine the 
association between cardio-metabolic biomarkers and SRH. Only few studies investigated this aspect. For instance, consistent with our findings, Tomten et al. [17] studied 18770 Norwegians and found increasing HDL raised the odds of reporting good SRH. Jylha et al. [16] studied 4065 elderly people aged 71 and over in the USA and found a significant association between biomarkers (blood levels of albumin, hemoglobin, HDL cholesterol and white cell count) and SRH after adjusting for age and sex. Moreover, Goodman et al. [15] studied 928 respondents aged 54 and over in Taiwan and found a significant association between SRH and clinical variables. The strongest association was found for HDL cholesterol among men even in the presence of other control variables.

This is as well the first study to ever evaluate the associations between SRH and inflammatory markers and measures of insulin resistance in any population and the first study to evaluate the levels and distribution of SRH, its determinants and its associations with biomarkers of cardio-metabolic disease among Chinese populations from different geographical areas and residence.

We considered the effect of comprehensive measurements of lifestyle, socio-demographic factors and presence of disease in the analysis. The extrapolation of our findings is somewhat limited due to the cross-sectional nature of our data and further longitudinal studies are required to confirm our findings. Additionally no clinical diagnostic was carried out to validate the result and the severity of the diseases. Also the functional and/or cognitive level of participants, which has been previously found to be associated with SRH [5], was not included in the analysis. Finally, although the sampling was conducted to represent the whole population of middle-aged/elderly Chinese, due to the limited number of selected sites ( 3 in each city) from only two major cities (Beijing and Shanghai) of China, the total population of China was not represented completely, hence limiting the potential extrapolation of our findings to alternative populations.

\section{Conclusion}

In general, we have found relatively low levels of good SRH in our population alongside with substantial impacts of area of residence, sleep quality and chronic diseases on SRH. Good SRH was associated with lower levels of inflammatory markers, insulin levels and insulin resistance, which might present SRH as a potential assessment tool to evaluate early deviations from health. Although further longitudinal studies are required to confirm our findings, interventions targeting social inequalities, sleep and physical activity patterns might not only contribute to prevent chronic morbidity but also to improve populations' perceived health.

\section{Abbreviations}

BMI: body mass index; CI: confidence interval; CVD: cardiovascular disease; DBP: diastolic blood pressure; HDL: high-density lipoprotein; LDL: low-density lipoprotein; MetS: metabolic syndrome; OR: odds ratio; SBP: systolic blood pressure; SD: standard deviation; SRH: self-rated health; TG: triglycerides; TCH: total cholesterol; RBP4: retinol-binding protein 4; ADIPO: adiponectin; IL-6: interleukin-6; PAI-1: plasminogen activator inhibitor-1; CRP: C-reactive protein; ANOVA: analysis of variance

\section{Competing interests}

The authors declare that they have no competing interests.

\section{Authors' contributions}

NHM performed the statistical analysis, interpretation of data and sequence alignment and drafted the manuscript. $\mathrm{AP}$ participated in design of the study, data collection and manuscript revision. XY, JW, QQ, YL, HL, ZY participated in the design of the study and data collection. XL carried out in design of the study and supervised the study progress in china. OHF conceived the study and participated in the analysis, drafting and revising the manuscript. All authors read and approved the final manuscript.

\section{Acknowledgements}

The authors would like to express their sincere appreciation to the study participants, the researchers and the healthcare professionals from the Centers for Disease Control and Prevention in Beijing and in Shanghai and also Dr. Gabrielle Osborne, Dr. Frans van der Ouderaa, Tony Dadd and Dr. Amir Ali Mohagheghi for their valuable comments.

This study was funded by grants SIBS2008006 from the Chief Scientist Program of Shanghai Institutes for Biological Sciences, Chinese Academy of Sciences to Dr. Xu Lin, KSCXI-YW-02 and KSCXI-YW-R-II 6 from the Knowledge Innovation Program of the Chinese Academy of Sciences, $\mathrm{CH}$ 2006-094I from the Shanghai-Unilever Research Development Fund.

\section{References}

I. Li ZB, Lam TH, Ho SY, Chan WM, Ho KS, Li MP, Leung GM, Fielding $\mathrm{R}$ : Age-versus time-comparative self-rated health in Hong Kong Chinese older adults. Int J Geriatr Psychiatry 2006, 2 I (8):729-739.

2. Lim WY, Ma S, Heng D, Bhalla V, Chew SK: Gender, ethnicity, health behaviour \& self-rated health in Singapore. BMC Public Health 2007, 7( I 47): 184

3. Singh-Manoux A, Gueguen A, Martikainen P, Ferrie J, Marmot M, Shipley $M$ : Self-rated health and mortality: short- and long-term associations in the Whitehall II study. Psychosom Med 2007, 69(2): $138-143$.

4. Idler EL, Benyamini Y: Self-rated health and mortality: a review of twenty-seven community studies. J Health Soc Behav 1997, 38(I):2I-37.

5. Bond ]: Self-rated health status as a predictor of death, functional and cognitive impairment: a longitudinal cohort study. Eur J Ageing 2006, 3:193-206.

6. Dowd JB, Zajacova A: Does the predictive power of self-rated health for subsequent mortality risk vary by socioeconomic status in the US? Int J Epidemiol 2007, 36(6): | 2 | 4- | 221.

7. Huisman $M$, van Lenthe $F$, Mackenbach J: The predictive ability of self-assessed health for mortality in different educational groups. Int J Epidemiol 2007, 36(6): I207-1213. 
8. Leung KK, Tang LY, Lue BH: Self-rated health and mortality in Chinese institutional elderly persons. J Clin Epidemiol 1997, 50(10): II07-III6.

9. Quesnel-Vallee A: Self-rated health: caught in the crossfire of the quest for 'true' health? Int J Epidemiol 2007, 36(6): I I6I-I I64.

10. Yu ES, Kean YM, Slymen DJ, Liu WT, Zhang M, Katzman R: Self-perceived health and 5-year mortality risks among the elderly in Shanghai, China. Am J Epidemiol 1998, I47(9):880-890.

II. Cheng ST, Chan AC: Social support and self-rated health revisited: is there a gender difference in later life? Soc Sci Med 2006, 63(I): $118-122$.

12. Cott CA, Gignac MA, Badley EM: Determinants of self rated health for Canadians with chronic disease and disability. J Epidemiol Community Health 1999, 53(I I):731-736.

13. Pei $X$, Rodriguez E: Provincial income inequality and selfreported health status in China during 199 I-7. J Epidemiol Community Health 2006, 60(1 2): I065-1069.

14. Yu LC, Zhang AY, Draper P, Kassab C, Miles T: Cultural correlates of self perceived health status among Chinese elderly. J Cross Cult Gerontol I997, I 2(I):73-89.

15. Goldman N, Glei DA, Chang MC: The role of clinical risk factors in understanding self-rated health. Ann Epidemiol 2004, I4(I):49-57.

16. Jylha M, Volpato S, Guralnik JM: Self-rated health showed a graded association with frequently used biomarkers in a large population sample. J Clin Epidemiol 2006, 59(5):465-47I.

17. Tomten SE, Hostmark AT: Self-rated health showed a consistent association with serum HDL-cholesterol in the cross-sectional Oslo Health Study. Int J Med Sci 2007, 4(5):278-287.

18. Pan A, Ye X, Franco OH, Li H, Yu Z, Wang J, Qi Q, Gu W, Pang X, Liu $H$, Lin $X$ : The association of depressive symptoms with inflammatory factors and adipokines in middle-aged and older chinese. PLOS ONE 2008, 3(I):el392.

19. YeX, Yu Z, Li H, Franco OH, Liu Y, Lin X: Distributions of C-reactive protein and its association with metabolic syndrome in middle-aged and older Chinese people. J Am Coll Cardiol 2007, 49(I7): I 798-1805.

20. Craig CL, Marshall AL, Sjostrom M, Bauman AE, Booth ML, Ainsworth BE, Pratt M, Ekelund U, Yngve A, Sallis JF, Oja P: International physical activity questionnaire: I 2-country reliability and validity. Med Sci Sports Exerc 2003, 35(8): I38I-I 395.

21. Zhou BF: Predictive values of body mass index and waist circumference for risk factors of certain related diseases in Chinese adults--study on optimal cut-off points of body mass index and waist circumference in Chinese adults. Biomed Environ Sci 2002, I 5(I):83-96.

22. Grundy SM, Cleeman JI, Daniels SR, Donato KA, Eckel RH, Franklin BA, Gordon DJ, Krauss RM, Savage PJ, Smith SC Jr, Spertus JA, Costa $F$ : Diagnosis and management of the metabolic syndrome: an American Heart Association/National Heart, Lung, and Blood Institute Scientific Statement. Circulation 2005 I I 2(I 7):2735-2752.

23. Radloff $L S$ : The CES-D scale: A self-report depression scale for research in the general population. Applied Psychological Measurement 1977, I:385-401.

24. Lin N: Measuring depressive symptomatology in China. J Nerv Ment Dis 1989, I77(3):|2|-|3|.

25. Zhang J, Norvilitis JM: Measuring Chinese psychological wellbeing with Western developed instruments. J Pers Assess 2002 79(3):492-5II.

26. Beekman AT, Penninx BW, Deeg DJ, Ormel J, Braam AW, van TW: Depression and physical health in later life: results from the Longitudinal Aging Study Amsterdam (LASA). J Affect Disord 1997, 46(3):219-231.

27. Pan A, Franco OH, Wang YF, Yu ZJ, Ye XW, Lin X: Prevalence and geographic disparity of depressive symptoms among middleaged and elderly in China. JAffect Disord 2008, 105(I-3):167-175.

28. Pan A, Ye X, Franco OH, Li H, Yu Z, Zou S, Zhang Z, Jiao S, Lin X: Insulin resistance and depressive symptoms in middle-aged and elderly Chinese: findings from the Nutrition and Health of Aging Population in China Study. J Affect Disord 2008, 109(12):75-82.

29. Qi Q, Yu Z, Ye X, Zhao F, Huang P, Hu FB, Franco OH, Wang J, Li H, Liu $Y$, Lin $X$ : Elevated retinol-binding protein 4 levels are associated with metabolic syndrome in Chinese people. I Clin Endocrinol Metab 2007, 92(I 2):4827-4834.
30. Levy JC, Matthews DR, Hermans MP: Correct homeostasis model assessment (HOMA) evaluation uses the computer program. Diabetes Care 1998, 2 I ( I 2):2191-2192.

31. Cheng YH, Chi I, Boey KW, Ko LS, Chou KL: Self-rated economic condition and the health of elderly persons in Hong Kong. Soc Sci Med 2002, 55(8): $14 \mid$ 5- 1424.

\section{Pre-publication history}

The pre-publication history for this paper can be accessed here:

http://www.biomedcentral.com/1471-2458/9/368/pre pub
Publish with Bio Med Central and every scientist can read your work free of charge

"BioMed Central will be the most significant development for disseminating the results of biomedical research in our lifetime. "

Sir Paul Nurse, Cancer Research UK

Your research papers will be:

- available free of charge to the entire biomedical community

- peer reviewed and published immediately upon acceptance

- cited in PubMed and archived on PubMed Central

- yours - you keep the copyright
BioMedcentral 